\title{
FEMALE PATIENTS WITH ANDROGENETIC ALOPECIA: IMMUNOHISTOCHEMICAL PICTURE OF SCALP BIOPSIES
}

\author{
Olena Salyenkova \\ Department of Infectious Diseases and Clinical Immunology \\ School of Medicine \\ V. N. Karazin Kharkiv National University \\ 4 Svobody sq., Kharkiv, Ukraine, 61022 \\ o.salenkova@karazin.ua
}

\begin{abstract}
The aim of the research. To expand the knowledge about immunohistochemical changes in female patients with androgenetic alopecia (AGA) focusing on non-androgenic co-factors of pathogenesis of the disease, as this may serve as a basis for improving therapeutic regimens.

Materials and methods. Scalp biopsies of female patients with androgenetic alopecia were examined by immunohistochemical method. The study included two groups: the main group of 30 female patients aged 22 to 40 years, average age $32.13 \pm 5.03$ years, and the control group that included 20 skin samples from women aged $25-40$ years (average age $34.75 \pm 4.19$ years) who underwent autopsies.

Results. It was found that AGA in women is characterized by a number of immunomorphological manifestations: the inflammatory infiltrate that consists of immunocompetent T-lymphocytes CD3+, CD4+ and CD8+, macrophages (CD68+); imbalance of growth polypeptides VEGF, TGF- $\beta 1$, EGFR; accumulation of oxidative stress enzymes eNOS and iNOS; accumulation of pathological fraction of Collagen IV.

Conclusions. The data obtained by this study helps to improve the concept of morphogenesis of AGA, and also can become a base to improve the standards of treatment of the disease. The pathological triade «oxidative stress-microinflammation- fibrosis» should be considered as a possible treatment target, as well as the imbalance of growth peptides.
\end{abstract}

Keywords: androgenetic alopecia, female patients, immunohistochemistry, inflammation, cytokines, growth factors, oxidative stress enzymes.

DOI: $10.21303 / 2504-5679.2021 .002043$

\section{Introduction}

Hair is an important part of our appearance and social communication. Hair loss could cause significant psychological trauma to both female and male patients $[1,2]$. This problem is further exacerbated by the limited treatment options available, which give only temporary results with many chances of side effects [3]. One of the main areas of research in trichology is the study of various aspects of AGA.

The aggressive effect of androgens on the hair follicle in androgen-dependent areas of the scalp under conditions of genetic predisposition is considered as the main etiopathogenetic mechanism of AGA $[4,5]$. However, recent studies show that oxidative stress, micro-inflammation, premature apoptosis and fibrosis can be considered as non-androgenic cofactors of its development [6].

The role of inflammation in the pathogenesis of AGA was suggested in the 90s of XX century [7]. Later, a number of studies reported about the inflammatory infiltration of activated T-cells and macrophages and moderate perifollicular fibrosis (which consisted of concentric layers of collagen) found in horizontal sections of scalp biopsies from areas of alopecia in women and men. The term «micro-inflammation» was proposed because the process was moderate and different from the destructive nature of classical inflammation [8].

Among the reasons that could initiate and participate in the further progression of AGA, oxidative stress is also considered. A number of independent studies have demonstrated the effect of oxidative stress on the induction of hair follicle cell apoptosis, which leads to an early transition to the catagen phase $[9,10]$. From an immunological point of view, catagen is a consequence of reduced expression of factors responsible for the maintenance of anagen - growth factors IGF-1, bFGF, VEGF. Also cytokines (including increased expression of TGF $\beta$, IL-1 $\alpha$ and TNF $\alpha$ ) are 
involved in this process as inducers of apoptosis. It was noted that the decrease in the proliferative activity of dermal papilla cells was associated with changes in cell morphology, including the expression of markers of oxidative stress and DNA damage [11].

But so far, the effects of oxidative stress, the inflammatory component and the fibrosis that has formed have not been studied properly. The elements of the molecular control of the immune system-induced pathological degeneration of the hair follicle by apoptosis-mediated organ death have not been studied as well.

Therefore, the aim of the research was to supplement information on the immunohistochemical (IHC) characteristics of the skin in women with AGA.

\section{Materials and methods}

The material for the study (main group) were biopsies of the scalp of women with AGA (30 patients aged 22 to 40 years, average age $32.13 \pm 5.03$ years), who were monitored in the period from 2017 to 2020 at the clinical base of the Department of Infectious Diseases and Clinical Immunology of the Medical Faculty of V. N. Karazin Kharkiv National University - «Trichology Institute». The control group included 20 skin samples from women aged 25-40 years (average age $34.75 \pm 4.19$ years) who underwent autopsies in the pathology department of Clinical city hospital No. 17 in Kharkiv. Tissue fragments were performed by standard histological methods, stained with hematoxylin and eosin.

During the study, the provisions of the Helsinki Declaration of the World Medical Association, the ethical code of the doctor of Ukraine and informing the patient about the nature of the study were strictly observed. All patients involved in the study signed informed consent to participate in the study. Protocol No. 2 of the Commission on Biomedical Ethics of V. N. Karazin Kharkiv National University of the Ministry of Science of Ukraine dated 13.05.2021.

IHC study was conducted on the basis of the laboratory of the Department of Pathological Anatomy of the Kharkiv Medical Academy of Postgraduate Education using antibodies and imaging system of Thermo scientific, Germany. The qualitative composition of the inflammatory infiltrate was studied using monoclonal antibodies (MCAB) to CD3, CD4 Clone 4B12, CD8 (SP16) (different fractions of T-lymphocytes, Ready-to-Use), CD 68 KP1 (macrophage marker, Ready-to-Use). To detect oxidative disorders, the expression of markers of nitric oxide metabolism was investigated: endothelial nitric oxide synthase (eNOS, Nitric Oxide Synthase, endothelial (eNOS), Rabbit Polyclonal Antibody at a dilution of 1:50) and inducible synthase of Nitric oxide (iNOS, Nitric oxide Synthase, inducible (iNOS), Rabbit Polyclonal Antibody at a dilution of 1: 100). The nature of angiogenesis was assessed by the expression of vascular endothelial growth factor (VEGF (VG1)). Epidermal growth factor expression was determined using EGFR Polyclonal Antibody (PCAB, titre 1:100). As a factor in the induction of the inflammatory process, transforming growth factor (PCAB to TGF- $\beta 1$ (V) Antibody, Ready-to-Use) was studied. Bcl 2 (124), Ready-to-Use, was used as a marker of apoptosis. Collagen IV (CIV22) with a titre of 1:50 was detected in the affected scalp areas.

Unmasking heat treatment was performed by boiling the sections in citrate buffer ( $\mathrm{pH}$ 6.0-7.0). UltraVision Quanto Detection Systems HRP Polymer (Thermo scientific, Germany) was used to visualize the primary antibodies. DAB (diaminobenzidine) was used as the chromogen.

The results were calculated using the Avtandilov eyepiece [12] in 10 randomly selected fields of view at a magnification of $\times 400$. The IHC markers were evaluated on two parameters: the degree of distribution and intensity of staining, taking into account the severity of the reaction and its location.

The degree of distribution of the marker was taken into account by the percentage of positively stained brown organelle cells from the total number of cells in the field of view. To assess the degree of colour intensity used a semi-quantitative scale: negative (0) was the reaction in the absence of staining of specific cell structures, weak positive (1+) was considered a reaction with weak or focal staining from 0 to $30 \%$ of cells, moderate positive $(2+)$ - when sufficient or focal staining of $30-60 \%$ of cells, pronounced or diffuse (3+) was considered a reaction when staining $60-90 \%$ of cells.

The complex of morphological studies was performed on a Primo Star microscope (Carl Zeiss) using the program AxioCam (ERc 5s). 


\section{Results}

Histopathological changes of the scalp in the main group of the study were quite characteristic, which allowed to morphologically confirm the clinical diagnosis. In all cases, the dermis was thinned with diffuse hair loss. The presence of hair of different diameters and vellus hair growing from miniaturized follicles were noticed. The hair bulb in the area of hair loss, in comparison with the control group, were located mainly in the reticular layer of the dermis with a significant reduction in the hypodermis. Between the non-affected hair follicles, in the area of empty hair bags in the area of the upper part of the hair follicles, areas of focal fibrosis of the dermis were found, they consisted of concentrically located bundles of connective tissue fibers. The number of sebaceous glands was increased compared to the skin of healthy women. In 28 cases out of 30 perivascularly, perifollicularly and between the acinus of the sebaceous glands, lympho-histiocytic infiltrate of varying severity was observed.

As a result of IHC examination of skin biopsies found the following. $76.6 \%$ of inflammatory infiltrate cells in the main group were represented by CD3+T-lymphocytes; $40 \%$ of them were CD4+T-lymphocytes, and they were observed mainly in the perifollicular zone between the acinuses of the sebaceous and sweat glands (Fig. 1). CD8+T-lymphocytes accounted for one third of all T-lymphocytes and were found in the connective tissue layers and perivascularly in the dermis (28 cases) and hypodermis (2 cases).

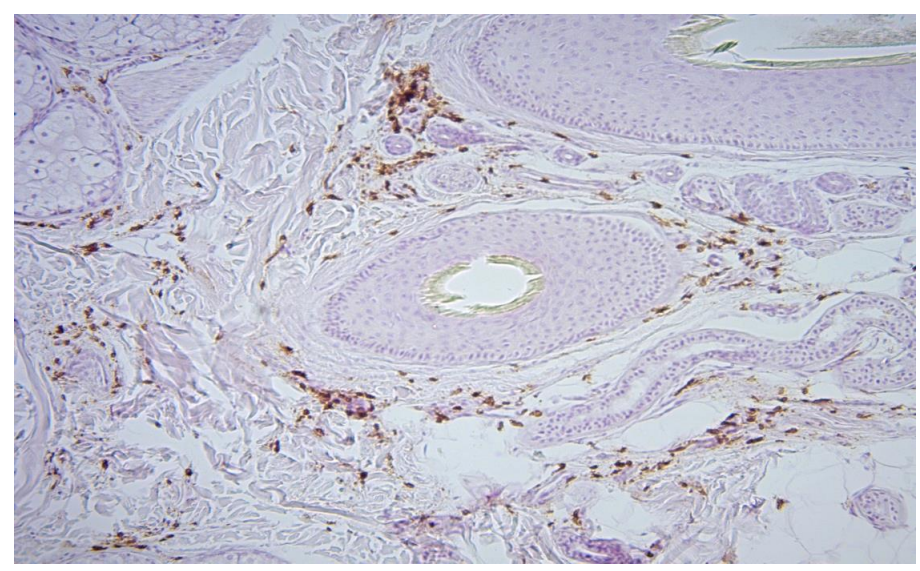

Fig. 1. CD4+T-lymphocytes in the inflammatory infiltrate of scalp biopsies of the main study group. Reaction from MCAB to CD4 Clone 4B12, $\times 100$

$11 \%$ of inflammatory infiltrate cells accounted for CD68+macrophages, which were located mainly in the fibrosing fields and in the upper parts of the dermis (Fig. 2).

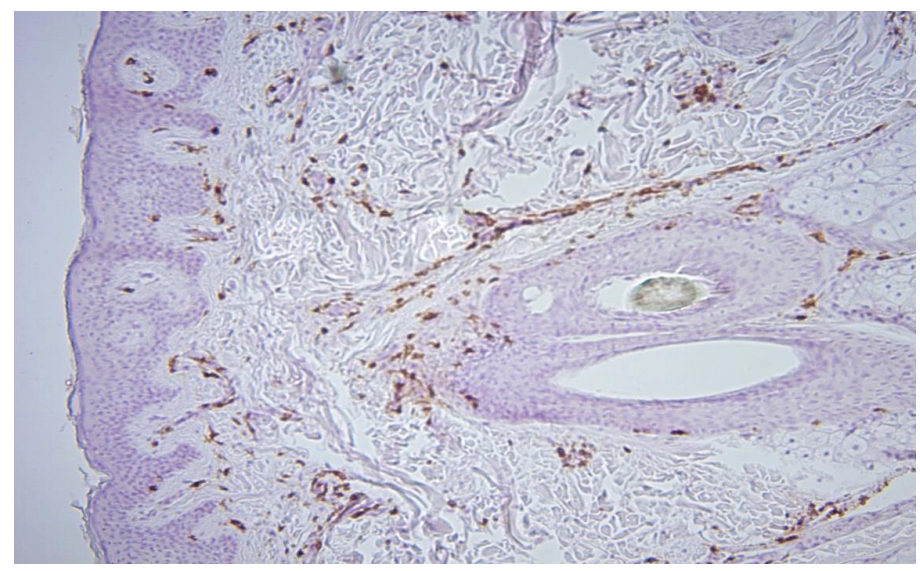

Fig. 2. Expressed macrophage infiltration in concentrically located fibrosing areas of scalp biopsies of the main study group. Reaction from MCAB to CD68 (CR1), ×100 
Expression of eNOS in the skin of healthy women was detected only in single macrophages, fibroblasts and vascular endotheliocytes, while in the main group it was observed in numerous macrophages, fibroblasts, endothelial and muscle layers of vessels, and outer root sheath keratinocytes (Fig. 3, a).

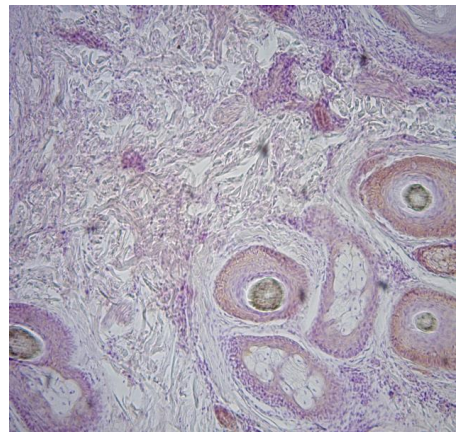

$a$

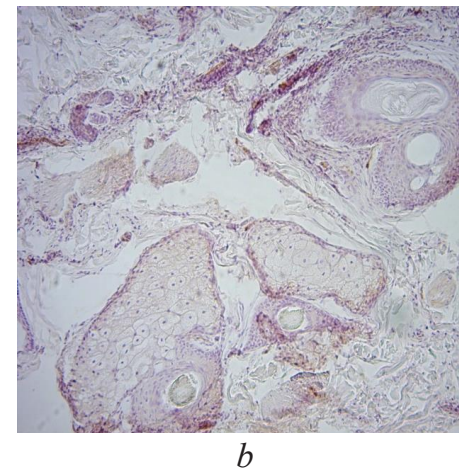

$b$

Fig. 3. Expression of eNOS and iNOS in the skin of healthy women: $a$ - Expression of eNOS in the outer root sheath. Reaction with PCAB to anti-eNOS; $b$ - Expression of iNOS

in endothelial cells and fibroblasts. Reaction with PCAB to anti-iNOS, $\times 100$

Inducible nitric oxide synthase iNOS was not determined in the control group, in scalp biopsies with AGA it was expressed in macrophages, vascular endotheliocytes, single epitheliocytes of follicles and sebaceous glands and in fibroblasts of the perifollicular fibrosing zone (Fig. 3, b).

The content of VEGF, as well as collagen type 4 was reduced: the reaction with vascular growth factor in the vascular endothelium was weak (1+), especially in areas of concentric fibrosis with depleted vascularization. The basal membranes of the epidermis, glands, hair follicles and blood vessels lost specialized type 4 collagen, and, conversely, its accumulation was noticed in atypical perifollicular fibrosing areas (Fig. 4).

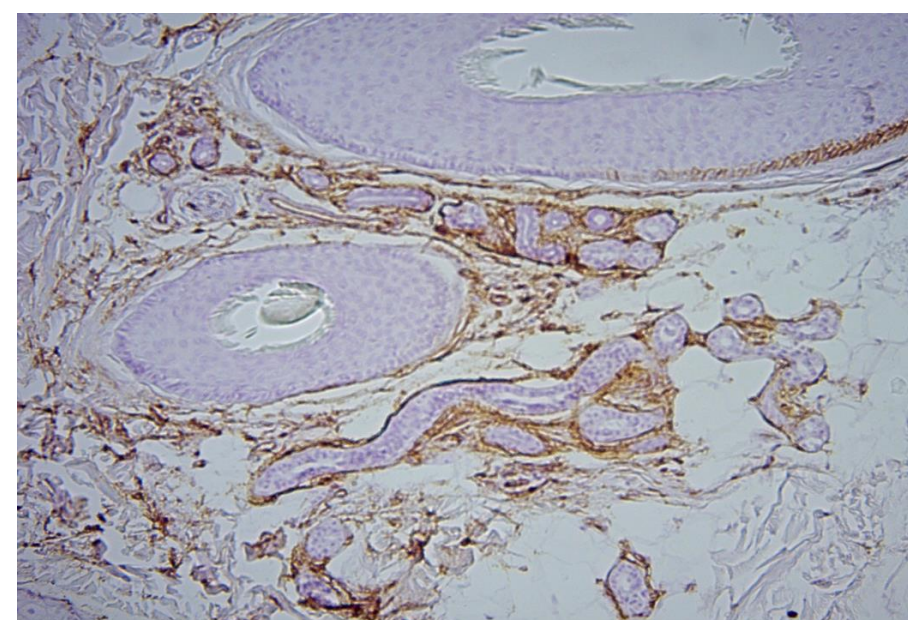

Fig. 4. Weak and intermittent expression of type 4 collagen in the basement membranes of skin structures of scalp biopsies of the main study group. Reaction from MCAB to Collagen IV (CIV22), $\times 100$

The content of TGF- $\beta 1$ and EGFR was increased in comparison with the control group. A strong positive reaction (3+) with PCAB to the transforming growth factor was observed above all in the numerous macrophages around the vessels and among the connective tissue strands of fibrosis (Fig. 5).

Epidermal growth factor marked both the thinned epidermis and the cells of the root sheath (Fig. 6). 


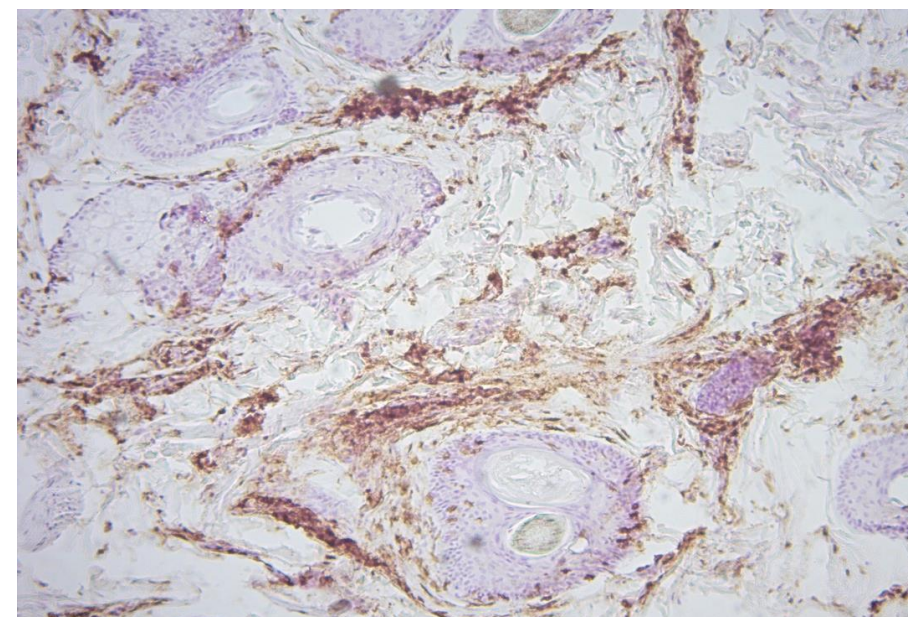

Fig. 5. Intensive expression of TGF- $\beta 1$ in skin macrophages of scalp biopsies of the main study group. Reaction with PCAB to TGF- $\beta 1 \mathrm{Ab}-3, \times 100$

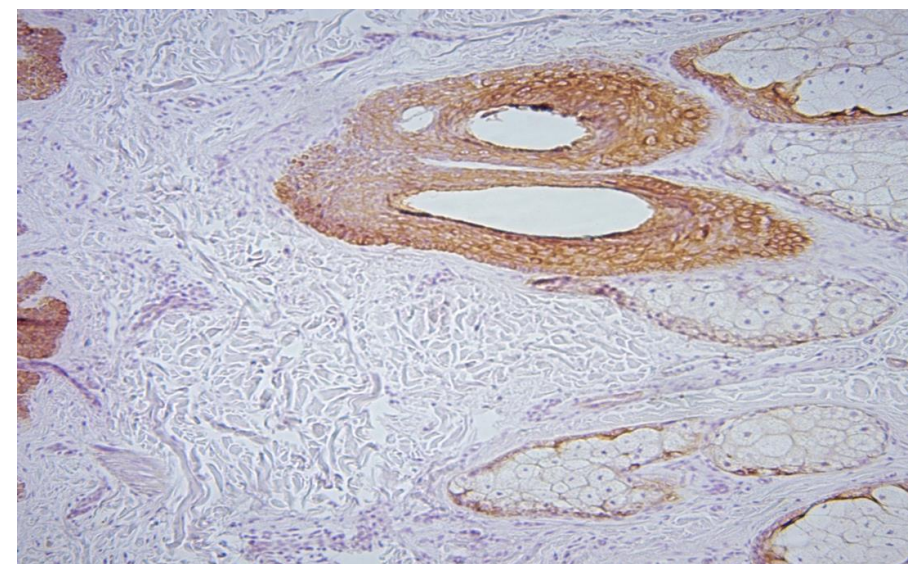

Fig. 6. Intensive expression of EGFR in the epithelial membranes of the hair follicle of the scalp biopsies of the main study group. Reaction with PCAB to EGFR Polyclonal Antibody, $\times 100$

The distribution of the antiapoptotic protein Bcl-2 was moderate and, more often, weakly expressed (1+), its expression was detected in inflammatory infiltrate cells and correlated with increased activity of nitric oxide synthase, especially inducible.

\section{Discussion}

The AGA process steadily leads to gradual hair loss in compromised areas of the scalp [13, 14]. The diagnostic program for AGA includes assessment of clinical manifestations of the disease, detection of pathology of ovarian and adrenal glands, pituitary gland, pancreas, thyroid gland, liver $[15,16]$ without detailed consideration of the immunomorphological condition of patients' skin. It is known that the main amount of regulatory proteins and growth factors, such as VEGF, TGF- $\beta 1$, EGFR, is synthesized in the dermal papilla [17]. Fibroblasts, epitheliocytes, endotheliocytes of its capillary in the inflammatory process, which was found in $93.3 \%$ of observations of the main study group, as well as T-lymphocytes and macrophages of inflammatory infiltrate lose the ability to fully produce these control substances [18]. In turn, the matrix of the hair follicle and the adjacent dermis begin to accumulate the products of enzymatic decomposition of nitric oxide eNOS and iNOS, which can be regarded as a manifestation of oxidative stress $[19,20]$. Type 4 collagen deposition is observed in the connective tissue fibers of the dermis and bundles of concentric perifollicular fibrosis. The complex of immunohistological changes that was identified during the study is a sign of pathological imbalance of the perifollicular environment and may be an inducer of premature apoptosis-mediated death of hair follicle stem cells, which was determined in all female 
patients with AGA. A kind of «vicious circle» is formed, which can be broken only by influencing each link of morphogenesis.

Immunocorrectives, anti-inflammatory drugs, antioxidants and antifibrosants are not currently included in the standards of AGA treatment. However, there is an assumption - whether their additional use will be a factor that improves patients' response to therapy.

Study limitations. The study included adult female patients with AGA of verying severity I-II stages according to the Ludwig scale.

Prospects for further research. The study of immunohistichemical changes in the affected area of scalp can be used to better understand immunological imbalance in patients with AGA and become a basis for improving treatment regimens.

\section{Conclusions}

AGA is accompanied by a number of immunomorphological changes in the skin, which can be detected by performing an IHC reaction. The features of the IHC reaction in the dermis of patients with AGA in the form of increased expression of nitric oxide synthases eNOS and iNOS indicate a violation of oxidative processes with an imbalance of immunocompetent T-lymphocytes CD3+, CD4+ and CD8+, macrophages (CD68+), growth polipeptides VEGF, TGF- $\beta 1$, EGFR. Determination of the pathological fraction of collagen type 4 can be considered as a marker of oxidative stress on chronic inflammation, which may be a predictor of further development of perifollicular fibrosis and disease progression. The data obtained by us allow to supplement the idea of the pathogenesis and morphogenesis of AGA.

\section{References}

[1] Piraccini, B. M., Alessandrini, A. (2014). Androgenetic alopecia. Giornale Italiano di Dermatologia e Venereologia: Organo Ufficiale, Societa Italiana di Dermatologia e Sifilografia, 149 (1), 15-24.

[2] Kelly, Y., Blanco, A., Tosti, A. (2016). Androgenetic Alopecia: An Update of Treatment Options. Drugs, 76 (14), $1349-1364$. doi: http://doi.org/10.1007/s40265-016-0629-5

[3] Lolli, F., Pallotti, F., Rossi, A., Fortuna, M. C., Caro, G., Lenzi, A. et. al. (2017). Androgenetic alopecia: a review. Endocrine, 57 (1), 9-17. doi: http://doi.org/10.1007/s12020-017-1280-y

[4] Kaliyadan, F., Nambiar, A., Vijayaraghavan, S. (2013). Androgenetic alopecia: An update. Indian Journal of Dermatology, Venereology, and Leprology, 79 (5), 613-625. doi: http://doi.org/10.4103/0378-6323.116730

[5] Sinclair, R., Torkamani, N., Jones, L. (2015). Androgenetic alopecia: new insights into the pathogenesis and mechanism of hair loss. F1000Research, 4, 585. doi: http://doi.org/10.12688/f1000research.6401.1

[6] Ovcharenko, Y., Salyenkova, O. (2021). Modern concept of the etiology and pathogenesis of androgenetic alopecia. The Journal of V. N. Karazin Kharkiv National University. Series «Medicine», 42, 92-100. doi: http://doi.org/10.26565/23136693-2021-42-11

[7] Magro, C. M., Rossi, A., Poe, J., Manhas-Bhutani, S., Sadick, N. (2011). The role of inflammation and immunity in the pathogenesis of androgenetic alopecia. Journal of Drugs in Dermatology, 10 (12), 1404-1411.

[8] Prie, B. E., Iosif, L., Tivig, I., Stoian, I., Giurcaneanu, C. (2016). Oxidative stress in androgenetic alopecia. Journal of medicine and life, 9 (1), 79-83.

[9] Rojas-Martínez, A., Martinez-Jacobo, L., Villarreal-Villarreal, C., Ortiz-López, R., Ocampo-Candiani, J. (2018). Genetic and molecular aspects of androgenetic alopecia. Indian Journal of Dermatology, Venereology and Leprology, 84 (3), $263-268$. doi: http://doi.org/10.4103/ijdvl.ijdvl_262_17

[10] Kaya Erdogan, H., Bulur, I., Kocaturk, E., Yildiz, B., Saracoglu, Z. N., Alatas, O. (2016). The role of oxidative stress in early-onset androgenetic alopecia. Journal of Cosmetic Dermatology, 16 (4), 527-530. doi: http://doi.org/10.1111/jocd.12300

[11] Chen, Q., Sun, T., Wang, J., Jia, J., Yi, Y., Chen, Y. (2019). Hydroxytyrosol prevents dermal papilla cells inflammation under oxidative stress by inducing autophagy. Journal of Biochemical and Molecular Toxicology, 33 (9). doi: http://doi.org/ $10.1002 / \mathrm{jbt} .22377$

[12] Avtandilov, G. G. (2002). Osnovy kolichestvennoi patologicheskoi anatomii. Moscow: Meditsina, 240.

[13] Varothai, S., Bergfeld, W. F. (2014). Androgenetic Alopecia: An Evidence-Based Treatment Update. American Journal of Clinical Dermatology, 15 (3), 217-230. doi: http://doi.org/10.1007/s40257-014-0077-5

[14] Ovcharenko, Y. S., Salyenkova, O. A. (2020). Analysis of expediency to use platelet-enriched plasma for treatment of androgenetic alopecia. International Medical Journal, 1, 64-67. doi: http://doi.org/10.37436/2308-5274-2020-1-14 
[15] Starace, M., Orlando, G., Alessandrini, A., Piraccini, B. M. (2020). Female Androgenetic Alopecia: An Update on Diagnosis and Management. American Journal of Clinical Dermatology, 21 (1), 69-84. doi: http://doi.org/10.1007/s40257-019-00479-x

[16] Rogers, N. (2013). Imposters of androgenetic alopecia: diagnostic pearls for the hair restoration surgeon. Facial Plastic Surgery Clinics of North America, 21 (3), 325-334. doi: http://doi.org/10.1016/j.fsc.2013.04.002

[17] Lu, G.-Q., Wu, Z.-B., Chu, X.-Y., Bi, Z.-G., Fan, W.-X. (2016). An investigation of crosstalk between Wnt/ $\beta$-catenin and transforming growth factor- $\beta$ signaling in androgenetic alopecia. Medicine, 95 (30), e4297. doi: http://doi.org/10.1097/ md.0000000000004297

[18] Inui, S., Itami, S. (2012). Androgen actions on the human hair follicle: perspectives. Experimental Dermatology, 22 (3), $168-171$. doi: http://doi.org/10.1111/exd.12024

[19] Pigeolet, E., Corbisier, P., Houbion, A., Lambert, D., Michiels, C., Raes, M. et. al. (1990). Glutathione peroxidase, superoxide dismutase, and catalase inactivation by peroxides and oxygen derived free radicals. Mechanisms of Ageing and Development, 51 (3), 283-297. doi: http://doi.org/10.1016/0047-6374(90)90078-t

[20] Upton, J. H., Hannen, R. F., Bahta, A. W., Farjo, N., Farjo, B., Philpott, M. P. (2015). Oxidative Stress-Associated Senescence in Dermal Papilla Cells of Men with Androgenetic Alopecia. Journal of Investigative Dermatology, 135 (5), $1244-1252$. doi: http://doi.org/10.1038/jid.2015.28

How to cite: Salyenkova, O. (2021). Female patients with androgenetic alopecia: immunohistochemical picture of scalp biopsies. EUREKA: Health Sciences, 5, 27-33. doi: http://doi.org/10.21303/2504-5679.2021.002043 